\title{
A New Analysis of Diffusion in Ternary Alloys: Application to f.c.c. Fe-Ni-Cr Alloys
}

\author{
I.V.Belova and G.E.Murch \\ Diffusion in Solids Group \\ School of Engineering \\ The University of Newcastle \\ Callaghan \\ New South Wales
}

Australia 2308

Keywords: bulk diffusion, iron alloys, interdiffusion, kinetics.

\begin{abstract}
In the present paper we develop new procedures for analysing chemical diffusion data in ternary alloys in order to provide ratios of atom-vacancy exchange frequencies. We base the procedures on the diffusion kinetics formalisms conceived by Manning and Moleko et al. As a reference point we also consider the simple formalism in which off-diagonal phenomenological coefficients are neglected. We apply the procedures to the measured interdiffusion coefficients in the $\gamma$-austenite phase of the $\mathrm{Fe}-\mathrm{Ni}-\mathrm{Cr}$ system at $1100 \mathrm{C}$. We show that the ratios of atom-vacancy exchange frequencies determined are reasonably consistent with those obtained from the tracer diffusion data using the same formalisms. We show that the three procedures give quite similar results for the frequency ratios in this alloy system where the atomic components have similar sizes and diffusivities.
\end{abstract}

\section{INTRODUCTION}

Because of the technological importance of ternary metallic alloys there have been numerous measurements of diffusion properties especially those involving interdiffusion [1]. Despite the large amount of data available, little has been done to extract from it any fundamental information such as jump frequency ratios and correlations in the random walks of the atoms. This is in contrast with the situation in other areas of diffusion such as dilute alloys where analysis is commonly made of both solute-enhanced solvent diffusivities to obtain atom-vacancy exchange frequency 
ratios or intrinsic diffusivities to obtain atom-vacancy exchange frequency ratios, see Ref.2.

Recently, the present authors were able to show that for the case of the multicomponent random alloy model simple and exact expressions exist for the ratios of the exchange frequencies in terms of the intrinsic diffusivities [3]. These expressions are also valid even when there is non-ideality as expressed (only) in the thermodynamic factors. The expressions have been used to analyse measured intrinsic diffusivities in a number of binary and ternary alloys [4]. However, because they require the rather tedious measurements of marker shifts, intrinsic diffusivities are not commonly measured in ternary alloys. The diffusivities generally measured are the four inter-diffusivities. They are typically analysed in the following way. First, the off-diagonal phenomenological coefficients are set equal to zero. This allows each of the six intrinsic diffusivities to be associated only with a single mobility factor (an ondiagonal phenomenological coefficient divided by the relevant composition) and a thermodynamic factor. Since the four inter-diffusivities themselves are expressible in terms of the intrinsic diffusivities, this allows their expression in terms of the mobility factors too. Development then of Darken-like expressions between the four interdiffusivities and the corresponding tracer diffusivities is straightforward; see, for example Refs. 1,5 and 6.

Manning [7] argued that the off-diagonal phenomenological coefficients should not be neglected in this way since they play an important role by carrying vacancy-wind or correlation information. In extreme cases the off-diagonal phenomenological coefficients may even predominate over the on-diagonal coefficients and can actually change the direction of an atomic flux. It is sometimes remarked that from an experimental point of view, vacancy-wind and correlation information may be implied in the on-diagonal coefficients (when the off-diagonal coefficients are set equal to zero). In other words, by setting the off-diagonal coefficients equal to zero, the on-diagonal coefficients are then forced to carry all the experimental diffusion information. This is a questionable procedure since it obscures information and confounds the theoretical meaning of the phenomenological coefficients.

Basing his analysis on the multi-component random alloy model in which the atomic species and the vacancy are randomly mixed and each atomic species $i$ exchanges with the vacancy with a frequency $\mathrm{w}_{\mathrm{i}}$, Manning [7] provided explicit 
expressions for the intrinsic diffusivities in terms of the corresponding tracer diffusivities and the geometrical tracer correlation factor $f_{0}$ for the lattice. These expressions, which included the effects of the off-diagonal coefficients, are of course easily extended to the inter-diffusivities. Importantly, it is seldom recognized that these expressions can also be reformulated in terms of ratios of the atom-vacancy exchange frequencies rather than the tracer diffusivities, see Ref. 8 for a related binary example, and Ref. 9 for a related ternary example. Reformulated expressions will be given in the present paper as a by-product of our analysis. Because Manning [7] was dealing with the random alloy model, thermodynamic ideality was assured and the thermodynamic factors were eliminated as well in the expressions. For disordered alloys, it is now generally recognized that non-ideality, which arises from the atomic interactions, should mainly affect the atom-vacancy exchange frequencies and the heat of solution. The correlation factors, the vacancy availability factors and the entropy of solution are relatively little affected see, for example, Refs 10,11. This means that it is reasonable to retain the thermodynamic factors in the expressions given by Manning [7]. Physically, it means that in the random alloy model the atomvacancy exchange frequencies, always conceived as averaged quantities [7,12], can be permitted to depend on composition using, say, the bond breaking model see, for example, Ref. 4.

A comprehensive self-consistent formalism for describing diffusion kinetics in the multi-component random alloy was developed by Moleko, Allnatt and Allnatt [13]. For brevity, this formalism will be referred to further in the present paper as 'MAA'. Recently, results from the formalism were extended and cast into a form more accessible for use in ternary alloys [9]. Extensive computer simulations of both tracer and collective correlation functions in the ternary random alloy were also reported in the same paper. It was shown that results of the Manning [12] formalism are only in semi-quantitative agreement with the simulation results whereas results of the MAA formalism are in almost exact agreement. It is clear that in general, the MAA formalism is the preferred one for describing diffusion kinetics in the ternary random alloy.

We mention here two other formalisms that have the potential to be used in analysing diffusion kinetics in ternary alloys. One is the Path Probability Method developed by Kikuchi and Sato [11] and refined a number of times [14,15] and the other is that developed recently by Nastar et al. [16]. The latter handles the basic 
diffusion correlation process in a rather more rigorous way than the former. Both these formalisms include the effects of interactions between the atoms at the outset, and, in this sense, have an advantage over the random alloy model in that they should be superior when analysing alloy systems that show pronounced short-range order. Furthermore, it would mean that in principle, the diffusion data could be analysed directly in terms of the more fundamental atomic interactions rather than ratios of atom-vacancy exchange frequencies. However, the calculations published so far have been confined to binary alloys and, furthermore, have employed fairly low-level statistical approximations to enable solutions to be obtained. The corresponding agreement with Monte Carlo results has so far only been semi-quantitative.

In the present paper we report on a comprehensive analysis based on the random alloy model using both the Manning [12] formalism and the MAA formalism. As a reference point we also consider the simple formalism wherein the off-diagonal phenomenological coefficients are neglected. We make use of the inter-diffusion data provided by Duh and Dayananda [17] and the tracer diffusion data provided by Rothman et al. [18] in the Fe-Ni-Cr austenite phase. Thermodynamic activity data are also included in the analysis in the form of thermodynamic factors. One purpose of the present paper is to show how these formalisms can be used in a practical sense for analysing inter-diffusion diffusion data in a ternary system to obtain ratios of atomvacancy exchange frequencies. Another purpose of the paper is to show that with respect to the exchange frequency ratios consistency is possible between the interdiffusion data and the tracer diffusion data. In section 2 we provide the theoretical development and strategy for the analysis and in section 3 we apply the analysis to the diffusion data in the $\gamma$ (f.c.c.) phase region of the $\mathrm{Fe}-\mathrm{Ni}-\mathrm{Cr}$ system.

\section{THEORY}

\subsection{Intrinsic diffusion coefficients are available}

In this paper, we use the standard notation where the integers 1, 2 and 3 represent the three atomic components of the ternary alloy and atomic component 3 is taken as the dependent concentration variable [1]. Let us consider the case when all six intrinsic diffusivities $\left(\mathrm{D}^{3}{ }_{11}, \mathrm{D}_{22}^{3}, \mathrm{D}^{3}{ }_{12}, \mathrm{D}^{3}{ }_{21}, \mathrm{D}^{3}{ }_{32}\right.$ and $\left.\mathrm{D}^{3}{ }_{31}\right)$ have been measured at a particular composition. In the multicomponent random alloy model Moleko and 
Allnatt [19] found a most useful exact relation connecting the $\mathrm{L}_{\mathrm{ij}}$, the phenomenological coefficients, and the atom-vacancy exchange frequencies. For the cubic ternary random alloy it is:

$$
\sum_{\mathrm{i}=1}^{3} \frac{\mathrm{L}_{\mathrm{ij}}}{\mathrm{w}_{\mathrm{i}}}=\frac{\mathrm{L}_{\mathrm{jj}}^{(0)}}{\mathrm{w}_{\mathrm{j}}}, \quad \forall \mathrm{j},
$$

where $\mathrm{L}_{\mathrm{jj}}^{(0)}=\mathrm{Nzc}_{\mathrm{V}} \mathrm{c}_{\mathrm{j}} \mathrm{w}_{\mathrm{j}} \mathrm{a}^{2} / 6 \mathrm{kT}$ is the uncorrelated phenomenological coefficient, where $w_{j}$ is the atom-vacancy exchange frequency with species $\mathrm{j}, \mathrm{N}$ is the number of sites per unit volume, $z$ is the coordination number, $c_{v}$ is the vacancy fraction, $c_{j}$ is the atomic fraction of species $\mathrm{j}$, $\mathrm{a}$ is the jump distance and $\mathrm{k}$ and $\mathrm{T}$ are the Boltzmann constant and absolute temperature respectively. In terms of the collective correlation factors (the correlated parts of the phenomenological coefficients) Eqn 1 is written as a sum-rule:

$$
\sum_{\mathrm{i}=1}^{3} \frac{\mathrm{f}_{\mathrm{ij}}^{(\mathrm{j})} \mathrm{w}_{\mathrm{j}}}{\mathrm{w}_{\mathrm{i}}}=1, \quad \forall \mathrm{j},
$$

where the collective correlation factors are defined by:

$$
\begin{gathered}
\mathrm{f}_{\mathrm{ii}} \equiv \frac{\mathrm{L}_{\mathrm{ii}}}{\mathrm{L}_{\mathrm{ii}}^{(0)}}, \\
\mathrm{f}_{\mathrm{ij}}^{(\mathrm{i})} \equiv \frac{\mathrm{L}_{\mathrm{ij}}}{\mathrm{L}_{\mathrm{ii}}^{(0)}}, \mathrm{i} \neq \mathrm{j} \\
\mathrm{f}_{\mathrm{ij}}^{(\mathrm{j})} \equiv \frac{\mathrm{L}_{\mathrm{ij}}}{\mathrm{L}_{\mathrm{jj}}^{(0)}}, \mathrm{i} \neq \mathrm{j}
\end{gathered}
$$

Eqn 1 leads to two explicit expressions for the ratios of the exchange frequencies in terms of the intrinsic diffusion coefficients at a given composition [3]:

$$
\begin{gathered}
\mathrm{w}_{1} / \mathrm{w}_{3}=\left(\mathrm{D}^{3}{ }_{11} \mathrm{D}_{22}^{3}-\mathrm{D}_{12}^{3} \mathrm{D}_{21}^{3}\right) /\left(\mathrm{D}^{3}{ }_{21} \mathrm{D}_{32}^{3}-\mathrm{D}^{3}{ }_{22} \mathrm{D}^{3}{ }_{31}\right) \\
\mathrm{w}_{2} / \mathrm{w}_{3}=\left(\mathrm{D}^{3}{ }_{11} \mathrm{D}^{3}{ }_{22}-\mathrm{D}^{3}{ }_{12} \mathrm{D}^{3}{ }_{21}\right) /\left(\mathrm{D}^{3}{ }_{12} \mathrm{D}^{3}{ }_{31}-\mathrm{D}^{3}{ }_{11} \mathrm{D}^{3}{ }_{32}\right)
\end{gathered}
$$


It should be noted that even in the presence of (thermodynamic) non-ideality these expressions remain unchanged [3]. These expressions make the analysis particularly straightforward. With the atom-vacancy exchange frequency ratios in hand, the tracer correlation factors and the collective correlation factors can then be readily obtained, if required, using either the Manning diffusion kinetics formalism or the MAA diffusion kinetics formalism, see Ref. 9.

\subsection{Interdiffusion coefficients are available.}

The processing of the four interdiffusion coefficients $\left(\widetilde{\mathrm{D}}_{11}^{3}, \widetilde{\mathrm{D}}_{12}^{3}, \widetilde{\mathrm{D}}_{21}^{3}\right.$, and $\left.\widetilde{\mathrm{D}}_{22}^{3}\right)$ to give ratios of the atom-vacancy exchange frequencies is rather more involved. First, as a preliminary, we note that expressions for the matrix of the intrinsic diffusion coefficients in the general (thermodynamically non-ideal) case are:

$$
\mathrm{D}=\mathrm{D}^{\prime} \mathrm{M}
$$

where the elements $d_{i j}^{\prime}(i=1,2,3 ; j=1,2)$ of the matrix $\mathbf{D}^{\prime}$ are:

$$
\begin{array}{lll}
\mathrm{d}_{11}^{\prime}=\frac{\mathrm{L}_{11}}{\mathrm{c}_{1}}-\frac{\mathrm{L}_{13}}{\mathrm{c}_{3}}, & \mathrm{~d}_{12}^{\prime}=\frac{\mathrm{L}_{12}}{\mathrm{c}_{2}}-\frac{\mathrm{L}_{13}}{\mathrm{c}_{3}}, & \mathrm{~d}_{21}^{\prime}=\frac{\mathrm{L}_{12}}{\mathrm{c}_{1}}-\frac{\mathrm{L}_{23}}{\mathrm{c}_{3}}, \\
\mathrm{~d}_{22}^{\prime}=\frac{\mathrm{L}_{22}}{\mathrm{c}_{2}}-\frac{\mathrm{L}_{23}}{\mathrm{c}_{3}}, & \mathrm{~d}_{31}^{\prime}=\frac{\mathrm{L}_{13}}{\mathrm{c}_{1}}-\frac{\mathrm{L}_{33}}{\mathrm{c}_{3}}, & \mathrm{~d}_{32}^{\prime}=\frac{\mathrm{L}_{23}}{\mathrm{c}_{2}}-\frac{\mathrm{L}_{33}}{\mathrm{c}_{3}} ;
\end{array}
$$

where the elements $m_{i j}(i=1,2 ; j=1,2)$ of the matrix $\mathbf{M}$ are:

$$
\begin{aligned}
& \mathrm{m}_{11}=\mathrm{c}_{1} \frac{\partial \mu_{1}}{\partial \mathrm{c}_{1}}, \quad \mathrm{~m}_{12}=\mathrm{c}_{2} \frac{\partial \mu_{2}}{\partial \mathrm{c}_{1}} \\
& \mathrm{~m}_{21}=\mathrm{c}_{1} \frac{\partial \mu_{1}}{\partial \mathrm{c}_{2}} \quad \mathrm{~m}_{22}=\mathrm{c}_{2} \frac{\partial \mu_{2}}{\partial \mathrm{c}_{2}}
\end{aligned}
$$

where $\mu_{\mathrm{i}}$ is the chemical potential of species $i$.

Since the interdiffusion coefficients $\widetilde{\mathrm{D}}_{\mathrm{ij}}^{3}, \mathrm{i}, \mathrm{j}=1,2$ can be expressed in terms of the intrinsic diffusion coefficients in the usual way, see, for example, Ref. 5: 


$$
\widetilde{\mathrm{D}}_{\mathrm{ij}}^{3}=\mathrm{D}_{\mathrm{ij}}^{3}-\mathrm{c}_{\mathrm{i}} \sum_{\mathrm{k}=1}^{3} \mathrm{D}_{\mathrm{kj}}^{3} \text {, where } \mathrm{i}, \mathrm{j}=1,2 \text {. }
$$

then it is straightforward to see that there is a relation analogous to Eqn 6:

$$
\widetilde{\mathrm{D}}=\widetilde{\mathrm{D}}^{\prime} \mathrm{M}
$$

where the elements $\widetilde{\mathrm{d}}_{\mathrm{ij}}^{\prime}$ of the matrix $\widetilde{\mathbf{D}}^{\prime}$ are related to the elements of the matrix $\mathbf{D}^{\prime}$ in the following way

$$
\widetilde{\mathrm{d}}_{\mathrm{ij}}^{\prime}=\mathrm{d}_{\mathrm{ij}}^{\prime}-\mathrm{c}_{\mathrm{i}} \sum_{\mathrm{k}=1}^{3} \mathrm{~d}_{\mathrm{kj}}^{\prime} \text {, where } \mathrm{i}, \mathrm{j}=1,2
$$

and the $\mathrm{d}_{\mathrm{ij}}^{\prime}$ are given in terms of the $\mathrm{L}_{\mathrm{ij}}$ by Eqns 7, 8. This feature of separating out the thermodynamic activity information will prove to be useful in our analysis.

At this point in our discussion, we introduce the collective correlation factors and atom-vacancy exchange frequencies by making use of the standard decomposition of the $\mathrm{L}_{\mathrm{ij}}$ into its correlated and uncorrelated parts Eqn 3. The $\widetilde{\mathrm{d}}_{\mathrm{ij}}^{\prime}$ now become:

$$
\frac{\widetilde{\mathrm{d}}_{11}^{\prime}}{\mathrm{L}_{11}^{(0)}}=\frac{\overline{\mathrm{w}}_{1}+\mathrm{c}_{1} \mathrm{w}_{31}}{\mathrm{c}_{1} \overline{\mathrm{w}}_{1}}-\mathrm{f}_{12} \frac{\mathrm{c}_{1} \overline{\mathrm{w}}_{2}+\overline{\mathrm{w}}_{1}\left(1-\mathrm{c}_{1}\right)}{\mathrm{c}_{1}^{2} \overline{\mathrm{w}}_{1} \overline{\mathrm{w}}_{2}}-\mathrm{f}_{13} \frac{\left(\mathrm{c}_{3} \overline{\mathrm{w}}_{1}+\mathrm{c}_{1}\right)\left(\overline{\mathrm{w}}_{1}+\mathrm{c}_{1} \mathrm{w}_{31}\right)}{\mathrm{c}_{1}^{2} \mathrm{c}_{3} \overline{\mathrm{w}}_{1}^{2}}+\mathrm{f}_{23} \frac{\mathrm{c}_{1} \mathrm{w}_{23}}{\mathrm{c}_{1} \mathrm{c}_{3} \overline{\mathrm{w}}_{1} \overline{\mathrm{w}}_{2}}
$$

$$
\begin{aligned}
& \frac{\widetilde{\mathrm{d}}_{12}^{\prime}}{\mathrm{L}_{11}^{(0)}}=\frac{\mathrm{w}_{32}}{\overline{\mathrm{w}}_{1}}+\mathrm{f}_{12} \frac{\overline{\mathrm{w}}_{1}+\mathrm{c}_{1} \mathrm{w}_{21}}{\mathrm{c}_{1} \mathrm{c}_{2} \overline{\mathrm{w}}_{1}^{2}}-\mathrm{f}_{13} \frac{\overline{\mathrm{w}}_{1}+\mathrm{c}_{1} \mathrm{w}_{31}}{\mathrm{c}_{1} \mathrm{c}_{3} \overline{\mathrm{w}}_{1}^{2}}+\mathrm{f}_{23} \frac{\mathrm{w}_{23}\left(\mathrm{c}_{3} \overline{\mathrm{w}}_{2}+\mathrm{c}_{2}\right)}{\mathrm{c}_{2} \mathrm{c}_{3} \overline{\mathrm{w}}_{1} \overline{\mathrm{w}}_{2}} \\
& \frac{\widetilde{\mathrm{d}}_{21}^{\prime}}{\mathrm{L}_{11}^{(0)}}=\frac{\mathrm{c}_{2} \mathrm{w}_{31}}{\mathrm{c}_{1} \overline{\mathrm{w}}_{1}}+\mathrm{f}_{12} \frac{\overline{\mathrm{w}}_{2}+\mathrm{c}_{2} \mathrm{w}_{12}}{\mathrm{c}_{1}^{2} \overline{\mathrm{w}}_{1} \overline{\mathrm{w}}_{2}}+\mathrm{f}_{13} \frac{\mathrm{c}_{2} \mathrm{w}_{13}\left(\mathrm{c}_{3} \overline{\mathrm{w}}_{1}+\mathrm{c}_{1}\right)}{\mathrm{c}_{1}^{2} \mathrm{c}_{3} \overline{\mathrm{w}}_{1}^{2}}-\mathrm{f}_{23} \frac{\overline{\mathrm{w}}_{2}+\mathrm{c}_{2} \mathrm{w}_{32}}{\mathrm{c}_{1} \mathrm{c}_{3} \overline{\mathrm{w}}_{1} \overline{\mathrm{w}}_{2}} ; \\
& \frac{\widetilde{\mathrm{d}}_{22}^{\prime}}{\mathrm{L}_{11}^{(0)}}=\frac{\overline{\mathrm{w}}_{2}+\mathrm{c}_{2} \mathrm{w}_{32}}{\mathrm{c}_{1} \overline{\mathrm{w}}_{1}}-\mathrm{f}_{12} \frac{\overline{\mathrm{w}}_{2}+\mathrm{c}_{2} \mathrm{w}_{12}}{\mathrm{c}_{1} \mathrm{c}_{2} \overline{\mathrm{w}}_{1}^{2}}+\mathrm{f}_{13} \frac{\mathrm{c}_{2} \mathrm{w}_{13}}{\mathrm{c}_{1} \mathrm{c}_{3} \overline{\mathrm{w}}_{1}^{2}}-\mathrm{f}_{23} \frac{\left(\mathrm{c}_{3} \overline{\mathrm{w}}_{2}+\mathrm{c}_{2}\right)\left(\overline{\mathrm{w}}_{2}+\mathrm{c}_{2} \mathrm{w}_{32}\right)}{\mathrm{c}_{1} \mathrm{c}_{2} \mathrm{c}_{3} \overline{\mathrm{w}}_{1} \overline{\mathrm{w}}_{2}}
\end{aligned}
$$

where for conciseness we have used the notation $\overline{\mathrm{w}}_{1}$ and $\overline{\mathrm{w}}_{2}$ for $\mathrm{w}_{1} / \mathrm{w}_{3}$ and $\mathrm{w}_{2} / \mathrm{w}_{3}, \mathrm{w}_{\mathrm{ij}}$ $=\left(\mathrm{w}_{\mathrm{i}}-\mathrm{w}_{\mathrm{j}}\right) / \mathrm{w}_{3}$ and $\mathrm{f}_{\mathrm{ij}}=\mathrm{c}_{\mathrm{i}} \mathrm{w}_{\mathrm{i}} \mathrm{f}_{\mathrm{ij}}{ }^{(\mathrm{i})}$. The 'sum-rule' (Eqns1 and 2) has also been used to 
arrive at Eqns 13-16. These relations can also be restated in terms of the interdiffusion coefficients and the $m_{i j}$ :

$$
\begin{aligned}
& \frac{\widetilde{\mathrm{D}}_{11}^{3}}{\mathrm{~L}_{11}^{(0)}}=\frac{\mathrm{m}_{11}\left(\overline{\mathrm{w}}_{1}+\mathrm{c}_{1} \mathrm{w}_{31}\right)+\mathrm{m}_{21} \mathrm{c}_{1} \mathrm{w}_{32}}{\mathrm{c}_{1} \overline{\mathrm{w}}_{1}}-\mathrm{f}_{12} \frac{\left(\overline{\mathrm{w}}_{1}+\mathrm{c}_{1} \mathrm{w}_{21}\right)\left(\mathrm{m}_{11} \mathrm{c}_{2} \overline{\mathrm{w}}_{1}-\mathrm{m}_{21} \mathrm{c}_{1} \overline{\mathrm{w}}_{2}\right)}{\mathrm{c}_{1}^{2} \mathrm{c}_{2} \overline{\mathrm{w}}_{1}^{2} \overline{\mathrm{w}}_{2}}- \\
& \mathrm{f}_{13} \frac{\left(\overline{\mathrm{w}}_{1}+\mathrm{c}_{1} \mathrm{w}_{31}\right)\left(\mathrm{m}_{11} \mathrm{c}_{3} \overline{\mathrm{w}}_{1}+\left(\mathrm{m}_{11}+\mathrm{m}_{21}\right) \mathrm{c}_{1}\right)}{\mathrm{c}_{1}^{2} \mathrm{c}_{3} \overline{\mathrm{w}}_{1}^{2}}+\mathrm{f}_{23} \frac{\mathrm{w}_{23}\left(\mathrm{~m}_{21} \mathrm{c}_{3} \overline{\mathrm{w}}_{2}+\left(\mathrm{m}_{21}+\mathrm{m}_{11}\right) \mathrm{c}_{2}\right)}{\mathrm{c}_{2} \mathrm{c}_{3} \overline{\mathrm{w}}_{1} \overline{\mathrm{w}}_{2}}
\end{aligned}
$$

$$
\begin{aligned}
& \frac{\widetilde{D}_{12}^{3}}{\mathrm{~L}_{11}^{(0)}}=\frac{\mathrm{m}_{22} \mathrm{c}_{1} \mathrm{w}_{32}+\mathrm{m}_{12}\left(\overline{\mathrm{w}}_{1}+\mathrm{c}_{1} \mathrm{w}_{31}\right)}{\mathrm{c}_{1} \overline{\mathrm{w}}_{1}}-\mathrm{f}_{12} \frac{\left(\overline{\mathrm{w}}_{1}+\mathrm{c}_{1} \mathrm{w}_{21}\right)\left(\mathrm{m}_{12} \mathrm{c}_{2} \overline{\mathrm{w}}_{1}-\mathrm{m}_{22} \mathrm{c}_{1} \overline{\mathrm{w}}_{2}\right)}{\mathrm{c}_{1}^{2} \mathrm{c}_{2} \overline{\mathrm{w}}_{1}^{2} \overline{\mathrm{w}}_{2}}- \\
& \mathrm{f}_{13} \frac{\left(\overline{\mathrm{w}}_{1}+\mathrm{c}_{1} \mathrm{w}_{31}\right)\left(\mathrm{m}_{12} \mathrm{c}_{3} \overline{\mathrm{w}}_{1}+\left(\mathrm{m}_{12}+\mathrm{m}_{22}\right) \mathrm{c}_{1}\right)}{\mathrm{c}_{1}^{2} \mathrm{c}_{3} \overline{\mathrm{w}}_{1}^{2}}+\mathrm{f}_{23} \frac{\mathrm{w}_{23}\left(\mathrm{~m}_{22} \mathrm{c}_{3} \overline{\mathrm{w}}_{2}+\left(\mathrm{m}_{12}+\mathrm{m}_{22}\right) \mathrm{c}_{2}\right)}{\mathrm{c}_{2} \mathrm{c}_{3} \overline{\mathrm{w}}_{1} \overline{\mathrm{w}}_{2}}
\end{aligned}
$$

$$
\begin{aligned}
& \frac{\widetilde{D}_{21}^{3}}{\mathrm{~L}_{11}^{(0)}}=\frac{\mathrm{m}_{11} \mathrm{c}_{2} \mathrm{w}_{31}+\mathrm{m}_{21}\left(\overline{\mathrm{w}}_{2}+\mathrm{c}_{2} \mathrm{w}_{32}\right)}{\mathrm{c}_{1} \overline{\mathrm{w}}_{1}}-\mathrm{f}_{12} \frac{\left(\overline{\mathrm{w}}_{2}+\mathrm{c}_{2} \mathrm{w}_{12}\right)\left(\mathrm{m}_{11} \mathrm{c}_{2} \overline{\mathrm{w}}_{1}-\mathrm{m}_{21} \mathrm{c}_{1} \overline{\mathrm{w}}_{2}\right)}{\mathrm{c}_{1}^{2} \mathrm{c}_{2} \overline{\mathrm{w}}_{1}^{2} \overline{\mathrm{w}}_{2}}+ \\
& \mathrm{f}_{13} \frac{\mathrm{c}_{2} \mathrm{w}_{13}\left(\mathrm{~m}_{11} \mathrm{c}_{3} \overline{\mathrm{w}}_{1}+\left(\mathrm{m}_{11}+\mathrm{m}_{21}\right) \mathrm{c}_{1}\right)}{\mathrm{c}_{1}^{2} \mathrm{c}_{3} \overline{\mathrm{w}}_{1}^{2}}-\mathrm{f}_{23} \frac{\left(\overline{\mathrm{w}}_{2}+\mathrm{c}_{2} \mathrm{w}_{32}\right)\left(\mathrm{m}_{21} \mathrm{c}_{3} \overline{\mathrm{w}}_{2}+\left(\mathrm{m}_{11}+\mathrm{m}_{21}\right) \mathrm{c}_{2}\right)}{\mathrm{c}_{1} \mathrm{c}_{2} \mathrm{c}_{3} \overline{\mathrm{w}}_{1} \overline{\mathrm{w}}_{2}}
\end{aligned}
$$

$$
\begin{aligned}
& \frac{\widetilde{D}_{22}^{3}}{\mathrm{~L}_{11}^{(0)}}=\frac{\mathrm{m}_{12} \mathrm{c}_{2} \mathrm{w}_{31}+\mathrm{m}_{22}\left(\overline{\mathrm{w}}_{2}+\mathrm{c}_{2} \mathrm{w}_{32}\right)}{\mathrm{c}_{1} \overline{\mathrm{w}}_{1}}-\mathrm{f}_{12} \frac{\left(\overline{\mathrm{w}}_{2}+\mathrm{c}_{2} \mathrm{w}_{12}\right)\left(\mathrm{m}_{22} \mathrm{c}_{1} \overline{\mathrm{w}}_{2}-\mathrm{m}_{12} \mathrm{c}_{2} \overline{\mathrm{w}}_{1}\right)}{\mathrm{c}_{1}^{2} \mathrm{c}_{2} \overline{\mathrm{w}}_{1}^{2} \overline{\mathrm{w}}_{2}}+ \\
& \mathrm{f}_{13} \frac{\mathrm{c}_{2} \mathrm{w}_{13}\left(\mathrm{~m}_{12} \mathrm{c}_{3} \overline{\mathrm{w}}_{1}+\left(\mathrm{m}_{12}+\mathrm{m}_{22}\right) \mathrm{c}_{1}\right)}{\mathrm{c}_{1}^{2} \mathrm{c}_{3} \overline{\mathrm{w}}_{1}^{2}}-\mathrm{f}_{23} \frac{\left(\overline{\mathrm{w}}_{2}+\mathrm{c}_{2} \mathrm{w}_{32}\right)\left(\mathrm{m}_{22} \mathrm{c}_{3} \mathrm{w}_{2}+\left(\mathrm{m}_{12}+\mathrm{m}_{22}\right) \mathrm{c}_{2}\right)}{\mathrm{c}_{1} \mathrm{c}_{2} \mathrm{c}_{3} \overline{\mathrm{w}}_{1} \overline{\mathrm{w}}_{2}}
\end{aligned}
$$

From Eqns 13-16 the following identity is valid, see also Philibert [20]:

$$
\mathrm{c}_{1} \mathrm{c}_{2}\left(\widetilde{\mathrm{d}}_{11}^{\prime}-\widetilde{\mathrm{d}}_{22}^{\prime}\right)=\mathrm{c}_{2}\left(1-\mathrm{c}_{2}\right) \widetilde{\mathrm{d}}_{12}^{\prime}-\mathrm{c}_{1}\left(1-\mathrm{c}_{1}\right) \widetilde{\mathrm{d}}_{21}^{\prime}
$$

or, in terms of $\widetilde{D}_{\mathrm{ij}}^{3}$ and the $\mathrm{m}_{\mathrm{ij}}$ :

$$
\begin{aligned}
& \mathrm{c}_{2}\left(\mathrm{c}_{1} \mathrm{~m}_{22}+\left(1-\mathrm{c}_{2}\right) \mathrm{m}_{12}\right) \widetilde{\mathrm{D}}_{11}^{3}-\mathrm{c}_{1}\left(\mathrm{c}_{2} \mathrm{~m}_{11}+\left(1-\mathrm{c}_{1}\right) \mathrm{m}_{21}\right) \widetilde{\mathrm{D}}_{22}^{3} \\
= & \mathrm{c}_{2}\left(\left(1-\mathrm{c}_{2}\right) \mathrm{m}_{11}+\mathrm{c}_{1} \mathrm{~m}_{21}\right) \widetilde{\mathrm{D}}_{12}^{3}-\mathrm{c}_{1}\left(\left(1-\mathrm{c}_{1}\right) \mathrm{m}_{22}+\mathrm{c}_{2} \mathrm{~m}_{12}\right) \widetilde{\mathrm{D}}_{21}^{3}
\end{aligned}
$$


This relation can be used as a criterion for the applicability of the ternary random alloy model to a particular material.

In our analysis so far, we have manipulated only standard definitions and the exact sum-rule relation (Eqns 1,2) for the ternary random alloy. We have four interdiffusion coefficients (only three of them are independent) and the thermodynamic factor matrix M. In effect, we have 3 equations and 6 unknown parameters $\left(\mathrm{w}_{1} / \mathrm{w}_{3}, \mathrm{w}_{2} / \mathrm{w}_{3}, \mathrm{f}_{12}, \mathrm{f}_{13}, \mathrm{f}_{23}\right.$ and $\mathrm{L}_{11}{ }^{(0)}$. $\mathrm{L}_{11}{ }^{(0)}$ cannot be obtained explicitly and is removed by taking ratios. To obtain the requisite extra equations we now need to make use of the Manning diffusion kinetics formalism or the MAA diffusion kinetics formalism. In the case of the Manning formalism we will have an extra four equations for $\mathrm{f}_{12}, \mathrm{f}_{13}, \mathrm{f}_{23}$ and $\mathrm{H}$ (the vacancy escape frequency function) in terms of $\mathrm{w}_{1} / \mathrm{w}_{3}$ and $\mathrm{w}_{2} / \mathrm{w}_{3}$. In the case of the MAA formalism, we will have a further eight equations for $\mathrm{f}_{12}, \mathrm{f}_{13}, \mathrm{f}_{23}, \mathrm{~g}_{1}, \mathrm{~g}_{2}, \mathrm{~g}_{3}$ (these are functions closely related to the offdiagonal collective correlation factors [9]), and $\mathrm{y}_{1}, \mathrm{y}_{2}$ (the latter are two auxiliary functions) in terms of $\mathrm{w}_{1} / \mathrm{w}_{3}$ and $\mathrm{w}_{2} / \mathrm{w}_{3}$.

\section{A. The Manning [7] formalism}

This formalism provides additional expressions for the collective correlation factors as follows:

$$
\mathrm{f}_{12}=\frac{2 \mathrm{c}_{1} \mathrm{c}_{2} \overline{\mathrm{w}}_{1} \overline{\mathrm{w}}_{2} \mathrm{H}}{\left(\mathrm{H}+2 \overline{\mathrm{w}}_{1}\right)\left(\mathrm{H}+2 \overline{\mathrm{w}}_{2}\right)} ; \mathrm{f}_{13}=\frac{2 \mathrm{c}_{1} \mathrm{c}_{3} \overline{\mathrm{w}}_{1} \mathrm{H}}{\left(\mathrm{H}+2 \overline{\mathrm{w}}_{1}\right)(\mathrm{H}+2)} ; \mathrm{f}_{23}=\frac{2 \mathrm{c}_{2} \mathrm{c}_{3} \overline{\mathrm{w}}_{1} \mathrm{H}}{\left(\mathrm{H}+2 \overline{\mathrm{w}}_{2}\right)(\mathrm{H}+2)},
$$

with the following equation for the function $\mathrm{H}$ :

$$
\frac{\mathrm{c}_{1} \overline{\mathrm{w}}_{1}}{\mathrm{H}+2 \overline{\mathrm{w}}_{1}}+\frac{\mathrm{c}_{2} \overline{\mathrm{w}}_{2}}{\mathrm{H}+2 \overline{\mathrm{w}}_{2}}+\frac{\mathrm{c}_{3}}{\mathrm{H}+2}=\frac{1-\mathrm{f}_{0}}{2} .
$$

\section{B. The MAA formalism.}

This formalism provides additional expressions for the collective correlation factors as follows:

$$
\mathrm{f}_{12}=-\frac{2 \overline{\mathrm{w}}_{1} \overline{\mathrm{w}}_{2} \mathrm{~g}_{1}}{\mathrm{c}_{\mathrm{v}}} ; \quad \mathrm{f}_{13}=-\frac{2 \overline{\mathrm{w}}_{1} \mathrm{~g}_{2}}{\mathrm{c}_{\mathrm{v}}} ; \quad \mathrm{f}_{23}=-\frac{2 \overline{\mathrm{w}}_{2} \mathrm{~g}_{3}}{\mathrm{c}_{\mathrm{v}}}
$$


and five equations for $g_{1}, g_{2}, g_{3}$ and the auxiliary functions $y_{1}$ and $y_{2}$ :

$$
\begin{gathered}
\mathrm{M}_{0} \mathrm{c}_{\mathrm{v}} \mathrm{y}_{1}\left(\mathrm{~g}-\mathrm{F}_{3}\right)=\mathrm{c}_{1} \mathrm{c}_{2} \mathrm{c}_{\mathrm{v}}+\mathrm{c}_{2}\left(\mathrm{~g}_{1}+\mathrm{g}_{2}\right)\left(2 \overline{\mathrm{w}}_{1}+\mathrm{M}_{0} \overline{\mathrm{w}}_{2} \mathrm{~F}_{2}\right)+\mathrm{c}_{1} \mathrm{~g}_{1}\left(2 \overline{\mathrm{w}}_{2}+\mathrm{M}_{0} \overline{\mathrm{w}}_{1} \mathrm{~F}_{1}\right) ; \\
\mathrm{M}_{0} \mathrm{c}_{\mathrm{v}} \mathrm{y}_{1}\left(\mathrm{~g}-\overline{\mathrm{w}}_{1} \mathrm{~F}_{1}\right)=-\mathrm{c}_{3} \mathrm{~g}_{1}\left(2 \overline{\mathrm{w}}_{2}+\mathrm{M}_{0} \mathrm{~F}_{3}\right)+\mathrm{c}_{2} \mathrm{~g}_{2}\left(2+\mathrm{M}_{0} \overline{\mathrm{w}}_{2} \mathrm{~F}_{2}\right) ; \\
M_{0} c_{v} y_{1}\left(-g+\bar{w}_{2} F_{2}\right)=c_{1} c_{3} c_{v}+c_{3}\left(g_{1}+g_{2}\right)\left(2 \bar{w}_{1}+M_{0} F_{3}\right)+c_{1} g_{2}\left(2+M_{0} \bar{w}_{1} F_{1}\right) \\
\mathrm{M}_{0} \mathrm{c}_{\mathrm{v}} \mathrm{y}_{2}\left(-\mathrm{g}+\mathrm{F}_{3}\right)=\mathrm{c}_{1} \mathrm{c}_{2} \mathrm{c}_{\mathrm{v}}+\mathrm{c}_{2} \mathrm{~g}_{1}\left(2 \overline{\mathrm{w}}_{1}+\mathrm{M}_{0} \overline{\mathrm{w}}_{2} \mathrm{~F}_{2}\right)+\mathrm{c}_{1}\left(\mathrm{~g}_{1}+\mathrm{g}_{3}\right)\left(2 \overline{\mathrm{w}}_{2}+\mathrm{M}_{0} \overline{\mathrm{w}}_{1} \mathrm{~F}_{1}\right) ;
\end{gathered}
$$

$$
\mathrm{M}_{0} \mathrm{c}_{\mathrm{v}} \mathrm{y}_{2}\left(-\mathrm{g}+\overline{\mathrm{w}}_{2} \mathrm{~F}_{2}\right)=-\mathrm{c}_{3} \mathrm{~g}_{1}\left(2 \overline{\mathrm{w}}_{1}+\mathrm{M}_{0} \mathrm{~F}_{3}\right)+\mathrm{c}_{1} \mathrm{~g}_{3}\left(2+\mathrm{M}_{0} \overline{\mathrm{w}}_{1} \mathrm{~F}_{1}\right)
$$

where $\mathrm{M}_{0}=2 \mathrm{f}_{0} /\left(1-\mathrm{f}_{0}\right)$ and

$$
\begin{gathered}
\mathrm{g}=1+\overline{\mathrm{w}}_{1}+\overline{\mathrm{w}}_{2}+2\left(\frac{\overline{\mathrm{w}}_{1}^{2}\left(\mathrm{~g}_{1}+\mathrm{g}_{2}\right)}{\mathrm{c}_{1} \mathrm{c}_{\mathrm{v}}}+\frac{\overline{\mathrm{w}}_{2}^{2}\left(\mathrm{~g}_{1}+\mathrm{g}_{3}\right)}{\mathrm{c}_{2} \mathrm{c}_{\mathrm{v}}}+\frac{\mathrm{g}_{2}+\mathrm{g}_{3}}{\mathrm{c}_{3} \mathrm{c}_{\mathrm{v}}}\right) \\
\mathrm{F}_{1}=1+2 \frac{\mathrm{g}_{1}\left(\overline{\mathrm{w}}_{1}-\overline{\mathrm{w}}_{2}\right)+\mathrm{g}_{1}\left(\overline{\mathrm{w}}_{1}-1\right)}{\mathrm{c}_{\mathrm{v}} \mathrm{c}_{1}} ; \mathrm{F}_{2}=1+2 \frac{\mathrm{g}_{1}\left(\overline{\mathrm{w}}_{2}-\overline{\mathrm{w}}_{1}\right)+\mathrm{g}_{3}\left(\overline{\mathrm{w}}_{2}-1\right)}{\mathrm{c}_{\mathrm{v}} \mathrm{c}_{2}} ; \\
\mathrm{F}_{3}=1+2 \frac{\mathrm{g}_{2}\left(1-\overline{\mathrm{w}}_{1}\right)+\mathrm{g}_{3}\left(1-\overline{\mathrm{w}}_{2}\right)}{\mathrm{c}_{\mathrm{v}} \mathrm{c}_{3}} .
\end{gathered}
$$

\section{Zero Off-Diagonal Phenomenological Coefficients}

We also give as a reference point the usual formalism wherein the off-diagonal phenomenological coefficients are simply put equal to zero. This is equivalent to putting $f_{12}=0, f_{13}=0$ and $f_{23}=0$. We then have two possibilities:

1. The solution of Eqns 13,14 and 16 with respect to $\overline{\mathrm{w}}_{1}$ and $\overline{\mathrm{w}}_{2}$ : 


$$
\overline{\mathrm{w}}_{1}=\frac{\mathrm{c}_{1}\left(\widetilde{\mathrm{d}}_{11}^{\prime}-\widetilde{\mathrm{d}}_{12}^{\prime}-\widetilde{\mathrm{d}}_{22}^{\prime} \mathrm{c}_{1}+\widetilde{\mathrm{d}}_{12}^{\prime} \mathrm{c}_{2}\right)}{\left(1-\mathrm{c}_{1}\right)\left(\widetilde{\mathrm{d}}_{12}^{\prime}+\widetilde{\mathrm{d}}_{22}^{\prime} \mathrm{c}_{1}-\widetilde{\mathrm{d}}_{12}^{\prime} \mathrm{c}_{2}\right)}, \quad \overline{\mathrm{w}}_{2}=1-\frac{\widetilde{\mathrm{d}}_{12}^{\prime}}{\widetilde{\mathrm{d}}_{12}^{\prime}+\widetilde{\mathrm{d}}_{22}^{\prime} \mathrm{c}_{1}-\widetilde{\mathrm{d}}_{12}^{\prime} \mathrm{c}_{2}}
$$

or in terms of $\widetilde{\mathrm{D}}_{\mathrm{ij}}^{3}$ (from Eqns 17-20):

$$
\begin{aligned}
& \overline{\mathrm{w}}_{1}=\frac{\mathrm{c}_{1} \mathrm{~m}_{11}\left(\widetilde{\mathrm{D}}_{11}^{3}\left(\mathrm{~m}_{11}-\mathrm{m}_{12}\right)+\widetilde{\mathrm{D}}_{12}^{3}\left(\mathrm{~m}_{21}-\mathrm{m}_{22}\right)\right)+\mathrm{c}_{1} \Delta_{\mathrm{m}}\left(\mathrm{c}_{1} \widetilde{\mathrm{D}}_{22}^{3}+\mathrm{c}_{2} \widetilde{\mathrm{D}}_{12}^{3}\right)}{\mathrm{c}_{3} \mathrm{~m}_{11} \mathrm{~m}_{22}\left(\widetilde{\mathrm{D}}_{11}^{3}+\widetilde{\mathrm{D}}_{12}^{3}\right)+\mathrm{c}_{1} \Delta_{\mathrm{m}}\left(\left(1-\mathrm{c}_{2}\right) \widetilde{\mathrm{D}}_{22}^{3}+\mathrm{c}_{2} \widetilde{\mathrm{D}}_{12}^{3}\right)} ; \\
& \overline{\mathrm{w}}_{2}=\frac{\mathrm{c}_{2}\left(\mathrm{~m}_{12}-\mathrm{m}_{11}\right)\left(\widetilde{\mathrm{D}}_{11}^{3} \mathrm{~m}_{12}+\widetilde{\mathrm{D}}_{12}^{3} \mathrm{~m}_{22}\right)+\mathrm{c}_{1} \Delta_{\mathrm{m}}\left(\left(1-\mathrm{c}_{1}\right) \widetilde{\mathrm{D}}_{22}^{3}+\mathrm{c}_{2} \widetilde{\mathrm{D}}_{12}^{3}\right)}{\mathrm{c}_{3} \mathrm{~m}_{11} \mathrm{~m}_{22}\left(\widetilde{\mathrm{D}}_{11}^{3}+\widetilde{\mathrm{D}}_{12}^{3}\right)+\mathrm{c}_{1} \Delta_{\mathrm{m}}\left(\left(1-\mathrm{c}_{2}\right) \widetilde{\mathrm{D}}_{22}^{3}+\mathrm{c}_{2} \widetilde{\mathrm{D}}_{12}^{3}\right)},
\end{aligned}
$$

where $\Delta_{\mathrm{m}}=\mathrm{m}_{11} \mathrm{~m}_{22}-\mathrm{m}_{12} \mathrm{~m}_{21}$.

2. The solution of Eqns 13,15 and 16 with respect to $\overline{\mathrm{w}}_{1}$ and $\overline{\mathrm{w}}_{2}$ :

$$
\overline{\mathrm{w}}_{1}=1-\frac{\widetilde{\mathrm{d}}_{21}^{\prime}}{\widetilde{\mathrm{d}}_{11}^{\prime} \mathrm{c}_{2}+\widetilde{\mathrm{d}}_{21}^{\prime}\left(1-\mathrm{c}_{1}\right)}, \quad \overline{\mathrm{w}}_{2}=\frac{\mathrm{c}_{2}\left(\widetilde{\mathrm{d}}_{22}^{\prime}-\widetilde{\mathrm{d}}_{21}^{\prime}\left(1-\mathrm{c}_{1}\right)-\widetilde{\mathrm{d}}_{11}^{\prime} \mathrm{c}_{2}\right)}{\left(1-\mathrm{c}_{2}\right)\left(\widetilde{\mathrm{d}}_{11}^{\prime} \mathrm{c}_{2}+\widetilde{\mathrm{d}}_{21}^{\prime}\left(1-\mathrm{c}_{1}\right)\right)} .
$$

or in terms of $\widetilde{\mathrm{D}}_{\mathrm{ij}}^{3}$ (from Eqns 17-20):

$$
\begin{aligned}
& \overline{\mathrm{w}}_{1}=\frac{\mathrm{c}_{1}\left(\mathrm{~m}_{21}-\mathrm{m}_{22}\right)\left(\widetilde{\mathrm{D}}_{21}^{3} \mathrm{~m}_{11}+\widetilde{\mathrm{D}}_{22}^{3} \mathrm{~m}_{21}\right)+\mathrm{c}_{2} \Delta_{\mathrm{m}}\left(\left(1-\mathrm{c}_{2}\right) \widetilde{\mathrm{D}}_{11}^{3}+\mathrm{c}_{1} \widetilde{\mathrm{D}}_{21}^{3}\right)}{\mathrm{c}_{3} \mathrm{~m}_{22}\left(\widetilde{\mathrm{D}}_{22}^{3} \mathrm{~m}_{21}+\widetilde{\mathrm{D}}_{21}^{3} \mathrm{~m}_{11}\right)+\mathrm{c}_{2} \Delta_{\mathrm{m}}\left(\left(1-\mathrm{c}_{2}\right) \widetilde{\mathrm{D}}_{11}^{3}+\mathrm{c}_{1} \widetilde{\mathrm{D}}_{21}^{3}\right)} \\
& \overline{\mathrm{w}}_{2}=\frac{\mathrm{c}_{2} \mathrm{~m}_{22}\left(\widetilde{\mathrm{D}}_{21}^{3}\left(\mathrm{~m}_{12}-\mathrm{m}_{11}\right)+\widetilde{\mathrm{D}}_{22}^{3}\left(\mathrm{~m}_{22}-\mathrm{m}_{21}\right)\right)+\mathrm{c}_{2} \Delta_{\mathrm{m}}\left(-\mathrm{c}_{2} \widetilde{\mathrm{D}}_{11}^{3}+\mathrm{c}_{1} \widetilde{\mathrm{D}}_{21}^{3}\right)}{\mathrm{c}_{3} \mathrm{~m}_{22}\left(\widetilde{\mathrm{D}}_{22}^{3} \mathrm{~m}_{21}+\widetilde{\mathrm{D}}_{21}^{3} \mathrm{~m}_{11}\right)+\mathrm{c}_{2} \Delta_{\mathrm{m}}\left(\left(1-\mathrm{c}_{2}\right) \widetilde{\mathrm{D}}_{11}^{3}+\mathrm{c}_{1} \widetilde{\mathrm{D}}_{21}^{3}\right)}
\end{aligned}
$$

\section{APPLICATION TO F.C.C. Fe-Ni-Cr ALLOYS}

The interdiffusivity data used in the present analysis were taken from the extensive measurements made by Duh and Dayananda [17], see also Dayananda [1], of the $\gamma$ (fcc) phase region of the Fe-Ni-Cr isotherm at $1100 \mathrm{C}$. The thermodynamic activity data for $\mathrm{Fe}, \mathrm{Ni}$ and $\mathrm{Cr}$ at $1100 \mathrm{C}$ were taken directly from the regular solution calculations provided by Duh and Dayananda [17]. These data together provided the input for the present analysis to obtain the ratios of the atom-vacancy exchange frequencies. Tracer diffusion data for $\mathrm{Fe}, \mathrm{Ni}$ and $\mathrm{Cr}$ at several compositions have been provided by Rothman et al. [18]. These were also analysed with the Manning and MAA formalisms to provide ratios of exchange frequencies. 
The system of equations to be solved in both the Manning and MAA formalisms is obviously non-linear. Then, for every particular composition we first need to estimate a possible solution. We assumed that the ratios of exchange

(a)

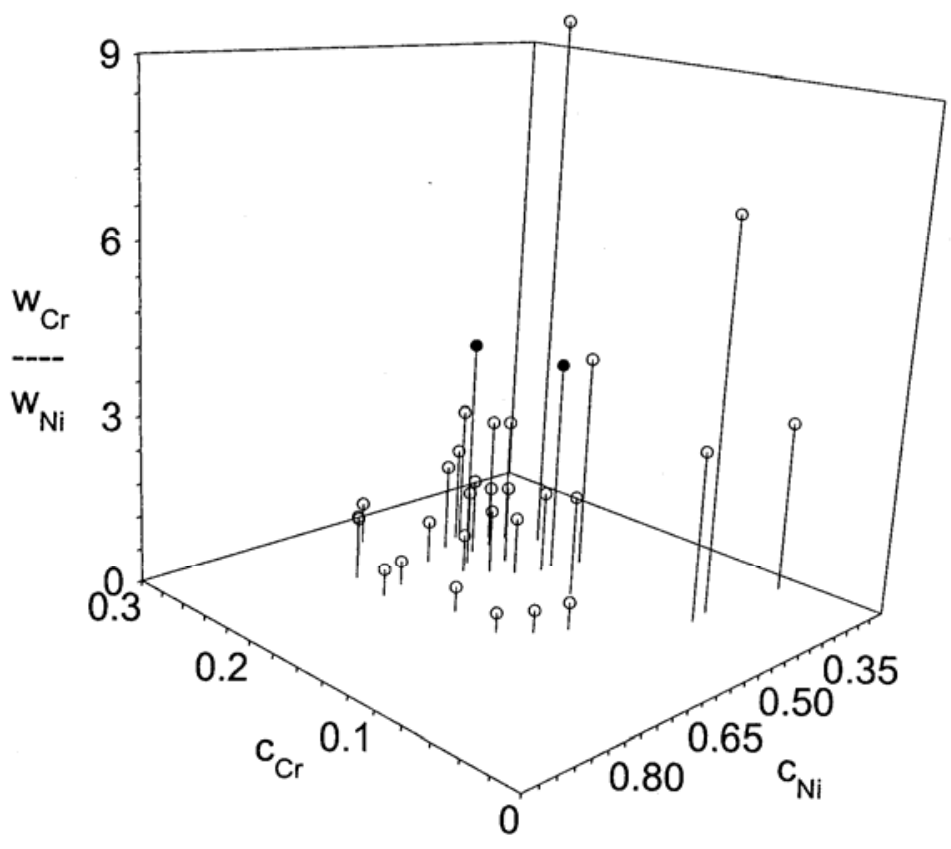

(b)

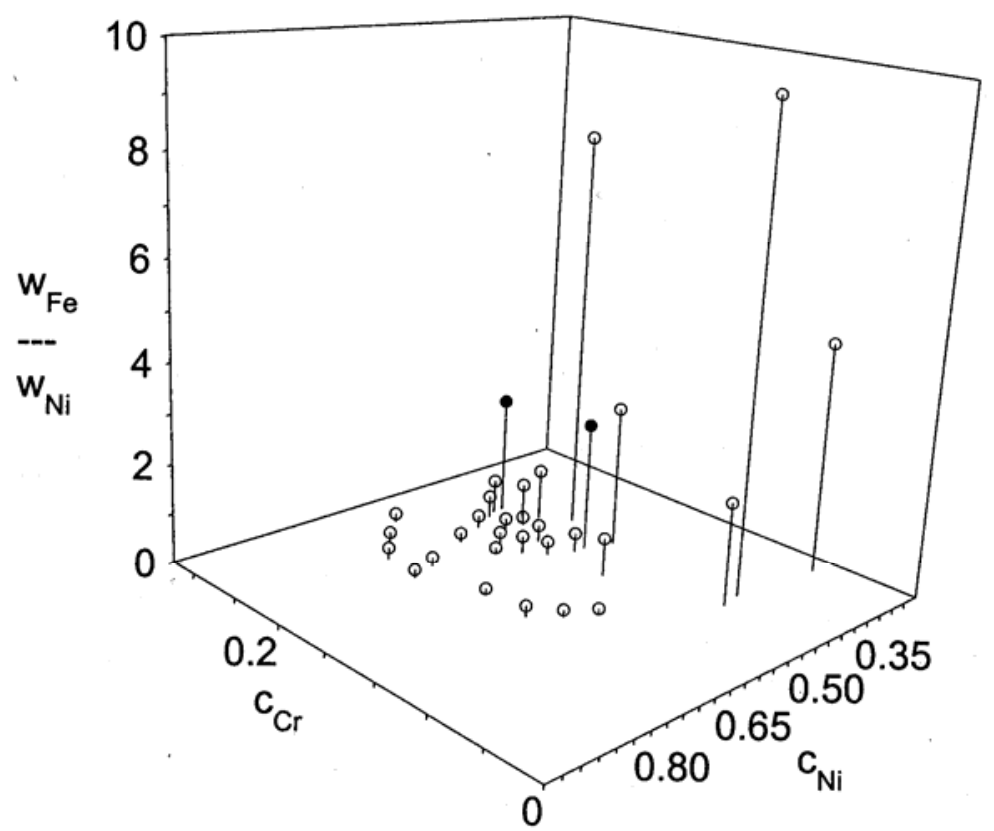


Figure $1 \mathrm{a}$, b. Ratios $\mathrm{w}_{\mathrm{Cr}} / \mathrm{w}_{\mathrm{Ni}}$ (a) and $\mathrm{w}_{\mathrm{Fe}} / \mathrm{w}_{\mathrm{Ni}}$ (b) as a function of atomic fractions $\mathrm{c}_{\mathrm{Cr}}$ and $\mathrm{c}_{\mathrm{Ni}}$. Open symbols - Manning analysis from interdiffusion and thermodynamic data, filled symbols - Manning analysis from tracer diffusion data.

(a)

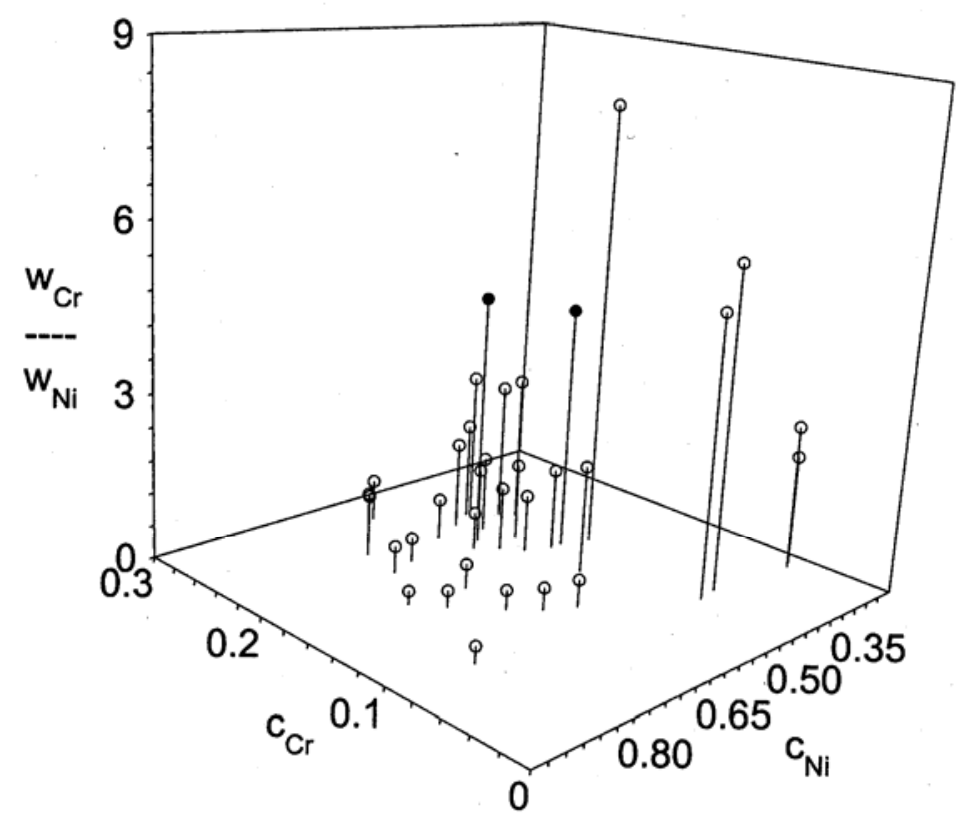

(b) 


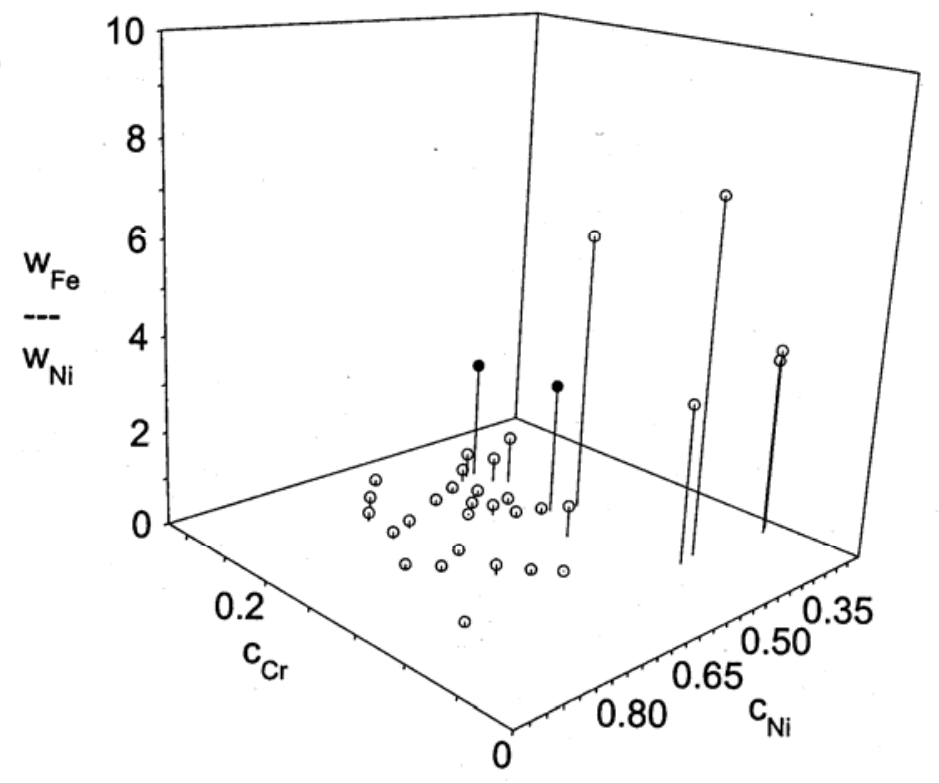

Figure 2 a, b. Ratios $\mathrm{w}_{\mathrm{Cr}} / \mathrm{w}_{\mathrm{Ni}}(\mathrm{a})$ and $\mathrm{w}_{\mathrm{Fe}} / \mathrm{w}_{\mathrm{Ni}}(\mathrm{b})$ as a function of atomic fractions $\mathrm{c}_{\mathrm{Cr}}$ and $\mathrm{c}_{\mathrm{Ni}}$. Open symbols - MAA analysis from interdiffusion and thermodynamic data, filled symbols - MAA analysis from tracer diffusion data.

frequencies could be bound between 0.01 and 100.0. Given the closeness of the tracer diffusion coefficients and the similarity in atomic size of the three species this is a very safe assumption. Then, in most cases, we could easily find a zeroth estimate for the solution using a graphical method. With this estimate in hand, we used the routine DNEQNF from the IMSL Library. This routine solves a system of nonlinear equations using a modified Powell hybrid algorithm and a finite-difference approximation to the Jacobian.

In about $25 \%$ of the interdiffusion data analysed with the Manning formalism no positive solution could be found for the exchange frequency ratios. In Fig 1a, b we present the results of the analysis using the Manning formalism. Open symbols represent our estimates of the ratios of the atom-vacancy exchange frequencies from the interdiffusion coefficients and filled symbols represent our estimates from the tracer diffusion coefficients. It is clear that the ratios tend to increase for higher $\mathrm{Ni}$ 


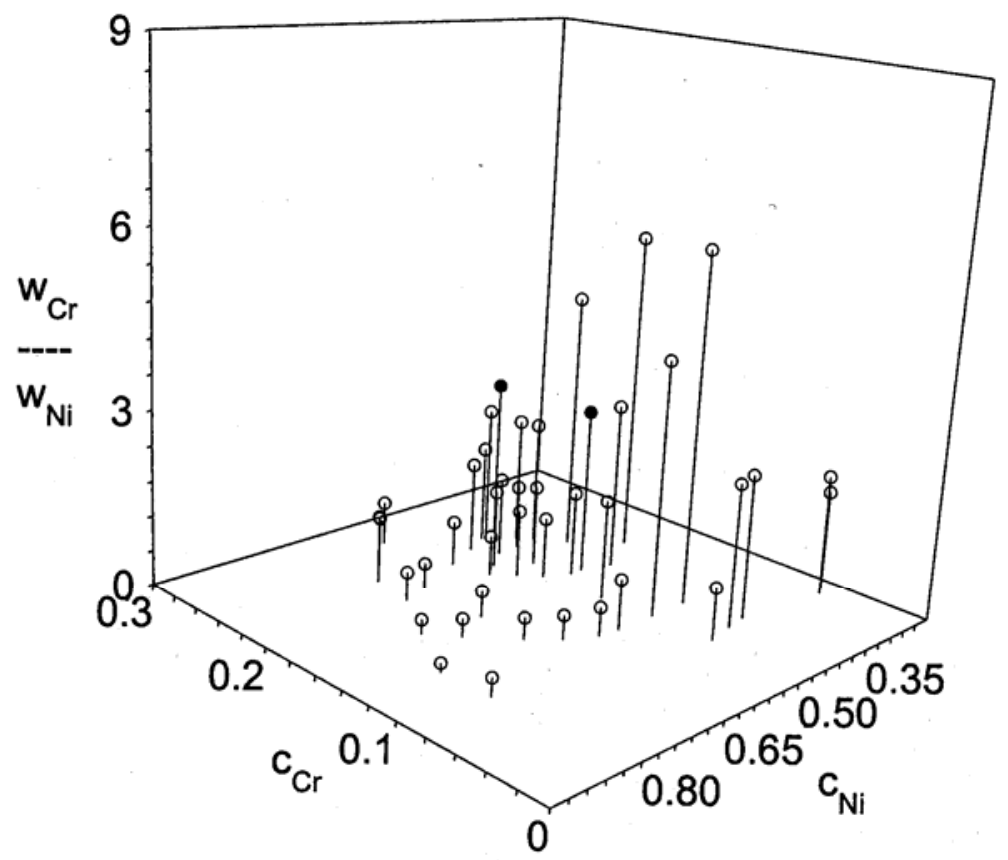

(b) 


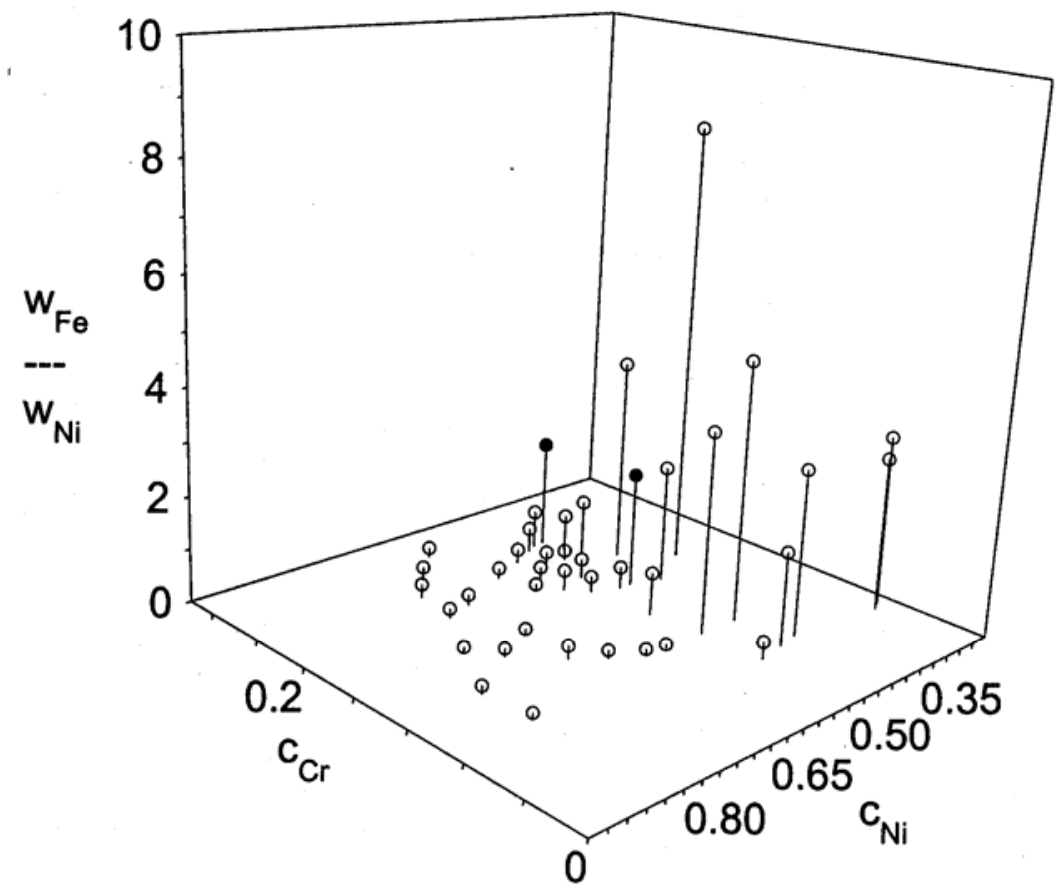

Figure $3 \mathrm{a}, \mathrm{b}$. Ratios $\mathrm{w}_{\mathrm{Cr}} / \mathrm{w}_{\mathrm{Ni}}$ (a) and $\mathrm{w}_{\mathrm{Fe}} / \mathrm{w}_{\mathrm{Ni}}$ (b) as a function of atomic fractions $\mathrm{c}_{\mathrm{Cr}}$ and $\mathrm{c}_{\mathrm{Ni}}$. Open symbols - 'zero off-diagonal phenomenological coefficient' analysis from interdiffusion and thermodynamic data, filled symbols - 'zero off-diagonal phenomenological coefficient' analysis from tracer diffusion data.

and $\mathrm{Cr}$ contents suggesting a significant effect from interactions. It is also clear that the frequency ratios derived from the tracer diffusion data are reasonably consistent with those derived from the interdiffusivity data. Such consistency had already been noted by Duh and Dayananda [17] using the analysis wherein the off-diagonal phenomenological coefficients are neglected.

In about $30 \%$ of the interdiffusivity data analysed using the MAA formalism no positive solution could be found for the exchange frequency ratios. In Fig $2 a, b$ we present the results of the analysis using the MAA formalism. Open symbols represent our estimates of the ratios of the exchange frequencies from the interdiffusion coefficients and filled symbols again represent our estimates from the tracer diffusion coefficients. The general behaviour of the frequency ratios is quite similar to that found above using the Manning analysis. Again, it is clear that the ratios tend to increase for higher $\mathrm{Ni}$ and $\mathrm{Cr}$ contents suggesting a significant effect from 
interactions. It is also clear that the frequency ratios derived from the tracer diffusion data are again reasonably consistent with those derived from the interdiffusivity data.

In Fig $3 a, b$ we present corresponding results for the ratios of the atom-vacancy exchange frequencies using the simple formalism wherein the off-diagonal phenomenological coefficients are put equal to zero. In about $11 \%$ of the interdiffusivity data analysed, no positive solution was obtained for the ratios. The pronounced similarity of the results of the exchange frequency ratios with those in the previous Figures from the Manning and MAA formalisms should be noted. It suggests that in an alloy such as $\gamma$ Fe-Ni-Cr where the atomic species have similar sizes and diffusivities it is entirely reasonable to neglect the off-diagonal phenomenological coefficients as a first approximation to obtaining the ratios of the atom-vacancy exchange frequencies.

We have mentioned above that positive solutions could not be obtained in some cases. This generally occurred for alloys fairly dilute in $\mathrm{Cr}$ thereby suggesting that $\mathrm{Cr}$ vacancy binding possibly occurs and the random alloy model is no longer appropriate. This is a well-known shortcoming of the random alloy model. In dilute fcc binary alloys, Manning [12] was able to show that if the ratio of the tracer diffusion coefficients exceeds about 5 then the random alloy is not appropriate (this is manifested as a ratio of exchange frequencies that is negative). The upper limit from MAA is similar. Determination of analogous limits for tracer and interdiffusivities in the ternary alloy would be useful and work in this direction is in progress by the present authors.

For reference purposes, in Tables $1-3$ (Appendix) we present the calculated ratios of the exchange frequencies. With the atom-vacancy exchange frequency ratios in hand, the tracer correlation factors and the collective correlation factors can then be readily obtained, if required, using either the Manning diffusion kinetics formalism or the MAA diffusion kinetics formalism, see Ref. 9.

We also performed an analysis of the uncertainties in the calculated exchange frequency ratios. Consider for example the ratios: $\widetilde{\mathrm{D}}_{\mathrm{ij}}^{3} / \widetilde{\mathrm{D}}_{11}^{3}$. Therefore, we have possible experimental errors combined in the following way (linear approximation): $\delta_{12}=\varepsilon_{12}-\varepsilon_{11}, \delta_{21}=\varepsilon_{21}-\varepsilon_{11}, \delta_{22}=\varepsilon_{22}-\varepsilon_{11}$, where $\varepsilon_{\mathrm{ij}}$ are the (relative) errors in the experimental $\widetilde{\mathrm{D}}_{\mathrm{ij}}^{3}$. Then, in usual way, we obtained a simple expression for the ratio of exchange frequencies $\left(\mathrm{w}_{1} / \mathrm{w}_{2}\right.$ and $\left.\mathrm{w}_{3} / \mathrm{w}_{2}\right)$ that is linear in $\delta_{\mathrm{ij}}$. This was done only for 
the 'zero off-diagonal coefficent' formalism. Calculation of the terms containing $\delta_{\mathrm{ij}}$ showed that in the majority of cases the errors for the exchange frequencies ratios are getting smaller than $\delta_{\mathrm{ij}}$; on average about a factor of \pm 0.5 . Accordingly, one can expect similar relative errors in the ratio of exchange frequencies to what there were in the original experimental data for the interdiffusivities.

In general, it is not possible to predict a priori the way in which each model would behave (in terms of the ratios of the exchange frequencies). We can only say that in the present case the terms in Eqns 13 - 16 containing cross correlations on average are all negative. Accordingly, when we employ the 'zero off-diagonal coefficient' formalism, we are effectively underestimating the 'true' interdiffusivities. Then we effectively underestimate the corresponding ratios of the exchange frequencies. This seems to be a general tendency. (Note, that we cannot estimate absolute values of exchange frequencies).

A suggested procedure for analysing the interdiffusivity data is to employ the 'zero off-diagonal coefficient' formalism first to obtain rough estimates of the ratios of the exchange frequencies. If the ratios are within a factor of two or so of unity then it would be appropriate to then use the Manning formalism to obtain better estimates of the ratios. Outside of this, and to obtain the most accurate ratios of the exchange frequencies, one should employ the almost exact MAA formalism [9].

\section{CONCLUSION}

In this paper, we have presented procedures for obtaining atom-vacancy exchange frequency ratios from measured interdiffusivity data in ternary alloys. We have based our procedures on the diffusion kinetics formalisms of Manning [12] and Moleko et al. [13]. Our reference point has been the simple formalism of neglecting off-diagonal phenomenological coefficients. We applied the procedures to fcc Fe-NiCr alloys at $1100 \mathrm{C}$. We were able to show that in these alloys (where the atomic sizes and diffusivities are similar) the ratios of exchange frequencies are fairly similar from the three formalisms and were reasonably consistent with those found from tracer diffusion data analyzed with the same formalisms.

\section{Acknowledgements}

We wish to thank the Australian Research Council for its support of this work in the Large Grants and Discovery Project Grants Schemes. One of us (IVB) wishes 
to thank the Australian Research Council for the award of a Queen Elizabeth II Fellowship.

\section{References}

[1] Dayananda MA. Diffusion in Solid Metals and Alloys. In: Mehrer H, editor, Landolt-Bornstein. New Series, Vol. 26. Berlin: Springer-Verlag, 1990. p.372.

[2] Allnatt AR and Lidiard AB. Atomic Transport in Solids. Cambridge: Cambridge University Press, 1993.

[3] Belova IV and Murch GE. Phil. Mag. Lett. 2001; 81: 661

[4] Belova IV and Murch GE. Defect Diffus. Forum 2002; 203/205: 125

[5] Kirkaldy JS and Young DJ. Diffusion in the Condensed State. London: Institute of Metals, 1987.

[6] Cserháti C, Ugaste U, van Dal MIH, Lousberg NJHGM, Kodentsov AA and van Loo FJJ. Defect Diffus. Forum 2001; 194-199: 189

[7] Manning JR. Metall. Trans. 1970; 504: 499

[8] Bakker H. In: Murch GE and Nowick AS, editors. Diffusion in Crystalline Solids. Orlando, Fl: Academic Press, 1984

[9] Belova IV, Allnatt AR and Murch GE. J Phys.: Condensed Matter, in press.

[10] Murch GE. Phil. Mag. A 1982; 45: 941

[11] Kikuchi R and Sato H. J. Chem. Phys. 1969; 51: 161

[12] Manning JR. Phys. Rev. B 1971; 4: 1111

[13] Moleko LK, Allnatt AR and Allnatt EL. Phil. Mag. A 1989; 59: 141.

[14] Sato H and Kikuchi R. Phys. Rev. B 1983; 28: 648

[15] Sato H, Ishikawa T and Kikuchi R. J. Phys. Chem. Solids 1985; 46: 1361

[16] Nastar M, Dobretsov VYu and Martin G. Phil. Mag. A 2000; 80: 155

[17] Duh JG and Dayananda MA. Diffus. Defect Data 1985; 39: 1

[18] Rothman SJ, Nowicki LJ and Murch GE. J. Phys. F 1980; 10: 383

[19] Moleko LK and Allnatt AR. Phil. Mag. A 1988; 58: 677

[20] Philibert J. Atom Movements. Diffusion and Mass Transport in Solids, Les Ulis: Editions de Physique, 1991.

\section{Appendix}

Table 1. Ratios $\mathrm{w}_{\mathrm{Cr}} / \mathrm{w}_{\mathrm{Ni}}(\mathrm{a})$ and $\mathrm{w}_{\mathrm{Fe}} / \mathrm{w}_{\mathrm{Ni}}(\mathrm{b})$ as a function of atomic fractions $\mathrm{c}_{\mathrm{Cr}}$ and $\mathrm{c}_{\mathrm{Ni}}$. Manning analysis from interdiffusion and thermodynamic data. (See Figure $1 \mathrm{a}$, b) 


\begin{tabular}{|c|c|c|c|}
\hline $\mathrm{C}_{\mathrm{cr}}$ & $\mathrm{C}_{\mathrm{Ni}}$ & $w_{C r} / w_{N i}$ & $w_{\mathrm{Fe}} / w_{\mathrm{Ni}}$ \\
\hline$\odot .228$ & 0.704 & 1.1577 & $\odot .2356$ \\
\hline$\odot .245$ & 0.666 & ๑. 8400 & 0.2320 \\
\hline ๑. 268 & $\odot .607$ & ๑. 7482 & $\odot .1647$ \\
\hline ๑. 199 & ๑.7155 & 0.4762 & 0.1546 \\
\hline ๑. 205 & 0.67 & ๑. 4265 & 0.1729 \\
\hline$\odot .22$ & ๑. 5805 & ๑. 7555 & 0.1830 \\
\hline ๑. 2295 & ๑. 5225 & 1.5783 & ๑. 2489 \\
\hline 0.238 & $\odot .48$ & 1.7115 & 0.4307 \\
\hline ๑. 242 & $\odot .461$ & 2.3973 & 0.6763 \\
\hline 0.158 & $\odot .6735$ & $\odot .4391$ & 0.1018 \\
\hline ๑. 196 & 0.565 & ๑. 6861 & ๑. .0932 \\
\hline$\odot .204$ & 0.538 & 1.3488 & 0.2336 \\
\hline ๑. 215 & 0.5 & 1.3730 & 0.2696 \\
\hline 0.111 & 0.638 & ๑. 3902 & 0.1349 \\
\hline ๑. 186 & 0.535 & 1.1594 & 0.3480 \\
\hline .216 & 0.461 & 1.1143 & 0.1572 \\
\hline ๑. 2255 & 0.4385 & 2.2822 & 0.6873 \\
\hline ๑. 1015 & ๑. 5935 & ๑. 4793 & 0.0968 \\
\hline$\odot .175$ & $\odot .51$ & 1.0475 & $\odot .2652$ \\
\hline ๑. 192 & ๑. 4865 & 1.4057 & 0.3347 \\
\hline 0.1335 & 0.5 & 1.8010 & 0.7793 \\
\hline 0.168 & 0.47 & 1.4786 & 0.3811 \\
\hline ๑ . 218 & ๑ . 4215 & 2.3019 & 1.0223 \\
\hline$\odot .064$ & 0.436 & 3.0627 & 2.0692 \\
\hline$\odot .162$ & 0.402 & 3.8812 & 2.8599 \\
\hline .202 & 0.388 & 9.6840 & 8.1061 \\
\hline$\odot .067$ & $\odot .398$ & 7.0257 & 9.6043 \\
\hline$\odot . \odot 6$ & 0.243 & 3.1375 & 4.7124 \\
\hline 0.124 & 0.678 & ๑. 3456 & 0.2117 \\
\hline
\end{tabular}

Table 2. Ratios $\mathrm{w}_{\mathrm{Cr}} / \mathrm{w}_{\mathrm{Ni}}(\mathrm{a})$ and $\mathrm{w}_{\mathrm{Fe}} / \mathrm{w}_{\mathrm{Ni}}(\mathrm{b})$ as a function of atomic fractions $\mathrm{c}_{\mathrm{Cr}}$ and $\mathrm{c}_{\mathrm{Ni}}$. MAA analysis from interdiffusion and thermodynamic data. (See Figure $2 \mathrm{a}, \mathrm{b}$ )

\begin{tabular}{llll}
\multicolumn{1}{c}{$\mathrm{C}_{\mathrm{Cr}}$} & \multicolumn{1}{c}{$\mathrm{C}_{\mathrm{Ni}}$} & \multicolumn{1}{c}{$\mathrm{W}_{\mathrm{Cr}} / \mathrm{W}_{\mathrm{Ni}}$} & $\mathrm{W}_{\mathrm{Fe}} / \mathrm{W}_{\mathrm{Ni}}$ \\
0.228 & 0.704 & 1.1598 & 0.1826 \\
0.245 & 0.666 & 0.8381 & 0.1884 \\
0.268 & 0.607 & 0.7464 & 0.1183 \\
0.199 & 0.7155 & 0.4743 & 0.1328 \\
0.205 & 0.67 & 0.4240 & 0.1502 \\
0.22 & 0.5805 & 0.7530 & 0.1151 \\
0.2295 & 0.5225 & 1.5867 & 0.0841 \\
0.238 & 0.48 & 1.7620 & 0.2626 \\
0.242 & 0.461 & 2.6390 & 0.5212 \\
0.0895 & 0.821 & 0.2932 & 0.1002 \\
0.158 & 0.6735 & 0.4377 & 0.0691 \\
0.196 & 0.565 & 0.6882 & 0.0060 \\
0.204 & 0.538 & 1.3528 & 0.0768 \\
0.215 & 0.5 & 1.3796 & 0.0910 \\
0.111 & 0.638 & 0.3871 & 0.0964
\end{tabular}




\begin{tabular}{|c|c|c|c|}
\hline 0.186 & 0.535 & 1.1665 & 0.2223 \\
\hline ๑. 2255 & 0.4385 & 2.5218 & 0.5270 \\
\hline$\odot .1015$ & $\odot .5935$ & $\odot .4854$ & 0.0012 \\
\hline$\odot .175$ & $\odot .51$ & 1.0485 & 0.1038 \\
\hline ๑. 192 & ๑. 4865 & 1. 4202 & 0.1295 \\
\hline 0.1335 & 0.5 & 1.9659 & 0.6832 \\
\hline$\odot .168$ & 0.47 & 1.4972 & 0.1149 \\
\hline$\odot .218$ & 0.4215 & 2.6717 & 1.0338 \\
\hline 0.064 & 0.436 & 5.1091 & 3.4183 \\
\hline 0.162 & $\odot .402$ & 8.0873 & 6.0285 \\
\hline 0.063 & $\odot .228$ & 2.0537 & 3.9819 \\
\hline 0.067 & ๑.398 & 5.8675 & 7.5277 \\
\hline$\odot .06$ & 0.243 & 2.6888 & 3.8294 \\
\hline 0.124 & 0.678 & 0.3444 & ○. 1987 \\
\hline$\odot .1465$ & 0.7355 & ๑. 2920 & 0.1224 \\
\hline$\odot .1625$ & $\odot .7705$ & ๑. 2292 & ๑. .0958 \\
\hline$\odot .268$ & 0.607 & 1.1575 & ๑. 6977 \\
\hline 0.205 & 0.67 & 0.5525 & 0.4932 \\
\hline$\odot .22$ & 0.5805 & ๑. 7985 & 0.1654 \\
\hline$\odot .158$ & 0.6735 & ๑. 6473 & 0.4434 \\
\hline
\end{tabular}

Table 3. Ratios $\mathrm{w}_{\mathrm{Cr}} / \mathrm{w}_{\mathrm{Ni}}(\mathrm{a})$ and $\mathrm{w}_{\mathrm{Fe}} / \mathrm{w}_{\mathrm{Ni}}(\mathrm{b})$ as a function of atomic fractions $\mathrm{c}_{\mathrm{Cr}}$ and $\mathrm{c}_{\mathrm{Ni}}$. 'Zero off-diagonal phenomenological coefficient' analysis from interdiffusion and thermodynamic data. (See Figure $3 \mathrm{a}, \mathrm{b}$ )

\begin{tabular}{llll}
\multicolumn{1}{c}{$\mathrm{C}_{\mathrm{Cr}}$} & \multicolumn{1}{c}{$\mathrm{C}_{\mathrm{Ni}}$} & $\mathrm{W}_{\mathrm{Cr}} / \mathrm{W}_{\mathrm{Ni}}$ & $\mathrm{W}_{\mathrm{Fe}} / \mathrm{W}_{\mathrm{Ni}}$ \\
0.228 & 0.704 & 1.1555 & 0.2463 \\
0.245 & 0.666 & 0.8428 & 0.2455 \\
0.268 & 0.607 & 0.7513 & 0.1749 \\
0.199 & 0.7155 & 0.4823 & 0.1644 \\
0.205 & 0.67 & 0.4364 & 0.1871 \\
0.22 & 0.5805 & 0.7599 & 0.1974 \\
0.2295 & 0.5225 & 1.5665 & 0.2643 \\
0.238 & 0.48 & 1.6808 & 0.4553 \\
0.242 & 0.461 & 2.3052 & 0.6976 \\
0.0895 & 0.821 & 0.2987 & 0.1159 \\
0.158 & 0.6735 & 0.4439 & 0.1095 \\
0.196 & 0.565 & 0.6888 & 0.1011 \\
0.204 & 0.538 & 1.3414 & 0.2498 \\
0.215 & 0.5 & 1.3631 & 0.2890 \\
0.111 & 0.638 & 0.4006 & 0.1496 \\
0.186 & 0.535 & 1.1528 & 0.3748 \\
0.216 & 0.461 & 1.1124 & 0.1713 \\
0.2255 & 0.4385 & 2.1897 & 0.7099 \\
0.1015 & 0.5935 & 0.4857 & 0.1079 \\
0.175 & 0.51 & 1.0460 & 0.2889 \\
0.192 & 0.4865 & 1.3905 & 0.3596 \\
0.1335 & 0.5 & 1.7151 & 0.8030 \\
0.168 & 0.47 & 1.4566 & 0.4096 \\
0.218 & 0.4215 & 2.1402 & 1.0195 \\
0.064 & 0.436 & 2.4729 & 1.7634
\end{tabular}




$\begin{array}{llll}0.162 & 0.402 & 2.8950 & 2.2231 \\ 0.202 & 0.388 & 4.5066 & 3.8695 \\ 0.063 & 0.228 & 1.7608 & 3.2692 \\ 0.18 & 0.321 & 5.6204 & 8.3985 \\ 0.06 & 0.485 & 0.8938 & 0.3143 \\ 0.067 & 0.398 & 2.4880 & 3.1247 \\ 0.06 & 0.243 & 2.1113 & 2.9301 \\ 0.10 & 0.559 & 0.8603 & 0.0716 \\ 0.124 & 0.678 & 0.3649 & 0.2350 \\ 0.1465 & 0.7355 & 0.3013 & 0.1444 \\ 0.1625 & 0.7705 & 0.2348 & 0.1087 \\ 0.124 & 0.825 & 0.1466 & 0.1410 \\ 0.10 & 0.424 & 6.0385 & 4.8753 \\ 0.10 & 0.49 & 4.3514 & 3.7704\end{array}$

